\title{
Analysis of a Feder
}

Ferenc Rádi

Msc student, BMGE (Budapest University of Technology and Economics)

Abstract - This paper covers an analysis in the cross section of sword fencing and the field of analytical mechanics and computer analysis. It aims to get answer to following questions in case of a normal blow with a feder on another feder: where are critical cross section/sections, where is the biggest stress, might the feder maybe break or not?

To inspect this question a model for the feder was created in a reliable, realistic, simple and closed form. Modeling a single blow acceleration and speed state at the chosen time will be calculated. Based on this input data equations and conclusions from the analytical mechanics were applied, where D'Alamberts principle is used. Results will be validated by finite element computer aided modeling and also applied on specified real life cases.

Keywords - feder (federschwert), model, collision, stress, break, blade-form, computer aided modeling

\section{FOREWORD}

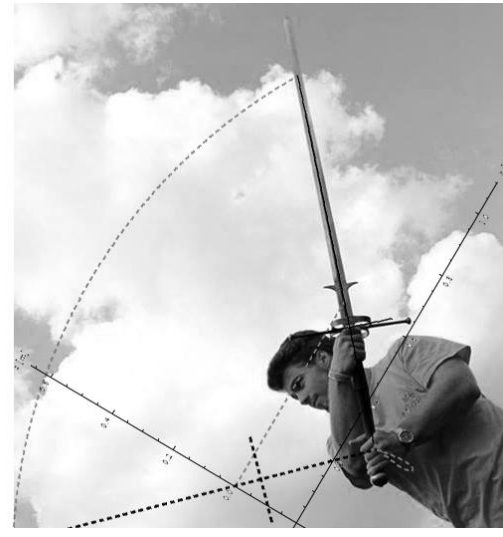

I created an article, which got special parameters for the readers. Who wants to read an article with a lot of equation, and explanation, will be disappointed. This is really not a scientists' way, but I think, I created a step, that everybody can take. You will see a lot of pictures, and mainly, from these you can understand the logic, and the result of the work. If you want more calculations, and difficult equations, then I offer you the documentation of the calculation ${ }^{1}$. If you have problems in this field, I created down here a link for some books².

\footnotetext{
${ }^{1}$ See for more: CALCULATION DOCUMENTATION

2 See for more: https://archive.org/details/mechanics032869mbp, or bttp://physicsdatabase.com/freephysics-books/
} 


\section{GROUND CONCEPTS}

\section{Velocity 3}

The velocity is a vector type quantity, which shows us the rate of change of the position of an object, and the direction of the change. When we want to describe the velocity vector $v$ of an object that has positions $\underline{x}(t)$ at time $t$ and $\underline{x}(t+d t)$ at time $t+d t$, can be computed as the derivative of position:

$$
\underline{v}=\lim _{d t \rightarrow 0} \frac{x(t+d t)-\underline{x}(\mathrm{t})}{d t}=\frac{d x}{d t}
$$

\section{Acceleration ${ }^{4}$}

The acceleration $\underline{a}$ is also a vector type quantity, and it describes the rate of velocity change of an object.

$$
\underline{a}=\frac{d v}{\overline{d t}}
$$

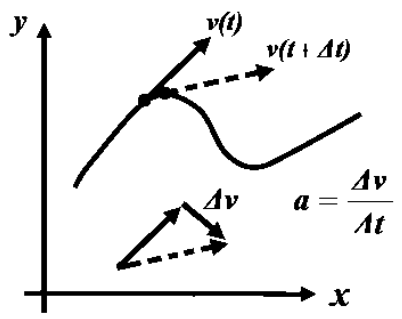

Rate of velocity change is acceleration ${ }^{5}$

\section{Angular velocity 6}

The angular velocity is a vector type quantity, it is defined as the rate of change of angular displacement $\underline{d \theta}$. The angular velocity $\underline{\omega}$, specifies the angular speed of an object and the axis about which the object is rotating.

$$
\underline{\omega}=\frac{d \theta}{\overline{d t}}
$$

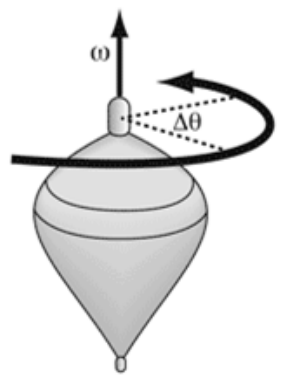

Angular velocity, in the case of a yoyo ${ }^{7}$

\footnotetext{
3 Source: László Holics: Physics

4 Source: László Holics: Physics

5 Source of the picture: http://upload.wikimedia.org/wikipedia/commons/5/56/Acceleration.JPG

${ }^{6}$ Source: László Holics: Physics
} 


\section{Angular acceleration 8}

Angular acceleration $\underline{\epsilon}$ is also a vector type quantity, it shows the rate of change of angular velocity. ${ }^{9}$

$$
\underline{\epsilon}=\frac{d \omega}{d t}
$$

\section{Rigid body}

In physics, a rigid body is an object with a special property: the distance between any two given points of a rigid body remains constant in time, which means the deformation is neglected. This mean this is only an idealization.

\section{Kinematical equation for rigid bodies}

These equations need a longer explanation ${ }^{10}$, I only write the summarized equations:

$$
\begin{gathered}
\underline{v_{A}}=\underline{v_{B}}+\underline{\omega} \times \underline{r_{A B}} \\
\underline{a_{A}}=\underline{a_{B}}+\underline{\epsilon} \times \underline{r_{A B}}+\underline{\omega} \times\left(\underline{\omega} \times \underline{r_{A B}}\right)
\end{gathered}
$$

where:

- $v_{A} / a_{A}$ is the speed/acceleration of point $\mathrm{A}$

- $v_{B} / a_{B}$ is the speed/acceleration of point $\mathrm{B}$

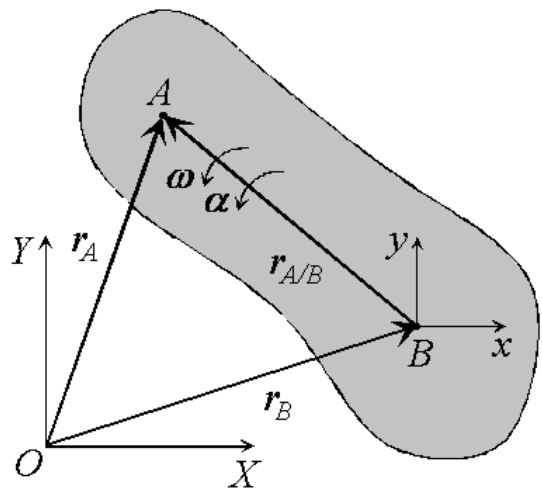

Kinematical equations $(\alpha=\epsilon)$

\section{Cross section}

In geometry, a cross section is the intersection of a body in a 3-dimensional space with a plane.

\section{Second moment of area}

The second moment of area, also known as moment of inertia of plane area, area moment of inertia, polar moment of area or second area moment, is a geometrical property of an area which reflects how its points are distributed with regard to an

7 Source of the picture: bttp:// wikipremed.com/01physicscards600/116a.gif

8 Source: László Holics: Physics

9 Source: László Holics: Physics

10 Source:

bttp://wmw.brown.edu/Departments/Engineering/Courses/En4/notes old/RigidKinematics/rigkin.btm 
arbitrary axis. The second moment of area is typically denoted with I. Its unit of dimension is length to fourth power. $\left(\mathrm{m}^{4}\right)$

It can be calculated from the following equations:

$$
\begin{aligned}
& I_{x}=\iint_{A} y^{2} d A \\
& I_{y}=\iint_{A} x^{2} d A
\end{aligned}
$$

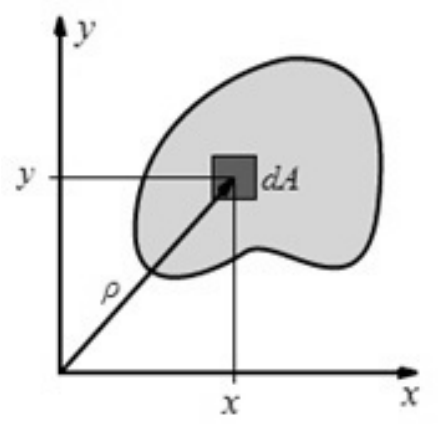

Second moment of area ${ }^{11}$

\section{Force 12}

In physics, a force is any influence that causes an object to undergo a certain change, either concerning its movement, direction, or geometrical form. In other words, a force can cause an object with mass to change its velocity (which includes to begin moving from a state of rest), i.e., to accelerate, or a flexible object to deform, or both. Force can also be described by intuitive concepts such as a push or a pull. A force has both magnitude and direction, making it a vector quantity. It is measured in the SI unit of Newtons and represented by the symbol F.

\section{Pressure ${ }^{13}$}

Pressure (symbol: $\mathrm{P}$ or $\mathrm{p}$ ) is the ratio of force to the area over which that force is distributed. Pressure is force per unit area applied in a direction perpendicular to the surface of an object. The SI unit of pressure is the newton per square metre, which is called the pascal $(\mathrm{Pa})$. A pressure of $1 \mathrm{~Pa}$ is small; it approximately equals the pressure exerted by a dollar bill resting flat on a table. Everyday pressures are often stated in kilopascals $(1 \mathrm{kPa}=1000 \mathrm{~Pa})$.

where:

$$
p=\frac{F}{A}
$$

- $F$ is the acting force over the area

- $A$ is the area, where F acts

11 Source of the picture: http://en.wikipedia.org/wiki/File:Polar Moment of Inertia.svg

12 Source: http://en.wikipedia.org/wiki/Force

13 Source: http://en.wikipedia.org/wiki/Pressure 


\section{Stress 14}

In mechanics, stress is a physical quantity that expresses the internal forces that neighbouring particles of a continuous material exert on each other. For example, when a solid vertical bar is supporting a weight, each particle in the bar pulls on the particles immediately above and below it. When a liquid is under pressure, each particle gets pushed inwards by all the surrounding particles, and, in reaction, pushes them outwards. These macroscopic forces are actually the average of a very large number of intermolecular forces and collisions between the particles in those molecules.

Stress inside a body may arise by various mechanisms, such as reaction to external forces applied to the bulk material (like gravity) or to its surface (like contact forces, external pressure, or friction). Any strain (deformation) of a solid material generates an internal elastic stress, analogous to the reaction force of a spring, that tends to restore the material to its original undeformed state.

The relation between mechanical stress, deformation, and the rate of change of deformation can be quite complicated, although a linear approximation may be adequate in practice if the quantities are small enough. Stress that exceeds certain strength limits of the material will result in permanent deformation (such as plastic flow, fracture, cavitation) or even change its crystal structure and chemical composition.

In some branches of engineering, the term stress is occasionally used in a looser sense as a synonym of "internal force". For example, in the analysis of trusses, it may refer to the total traction or compression force acting on a beam, rather than the force divided by the area of its cross-section.
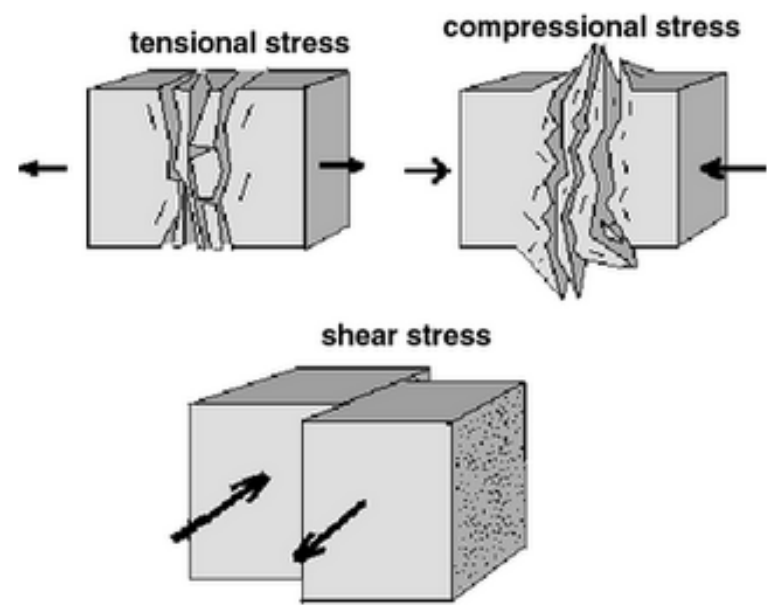

Various mechanical stresses 15

14 Source: http:// en.wikipedia.org/wiki/Stress (mechanics)

15 Source of the picture: http://img535.imageshack.us/img535/2152/physpic1.png 


\section{Stress in beams from bending 16}

In the Euler-Bernoulli theory of slender beams, a major assumption is that 'plane sections remain plane'. In other words, any deformation due to shear across the section is not accounted for (no shear deformation). Also, this linear distribution is only applicable if the maximum stress is less than the yield stress of the material. For stresses that exceed yield, refer to article plastic bending. At yield, the maximum stress experienced in the section (at the furthest points from the neutral axis of the beam) is defined as the flexural strength.

The Euler-Bernoulli equation for the quasistatic bending of slender, isotropic, homogeneous beams of constant cross-section under an applied transverse load $\mathrm{q}(\mathrm{x})$ is:

where:

$$
E I \frac{d w(x)}{d x}=q(x)
$$

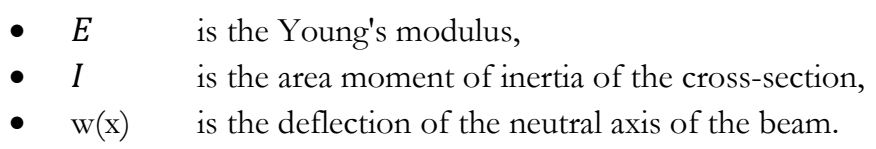

\section{Collision ${ }^{17}$}

A collision is an isolated event in which two or more moving bodies or cars (colliding bodies) exert forces on each other for a relatively short time. Although the most common colloquial use of the word "collision" refers to accidents in which two or more objects collide, the scientific use of the word "collision" implies nothing about the magnitude of the forces.

\section{D'Alembert's principle 18}

D'Alembert's principle, also known as the Lagrange-d'Alembert principle, is a statement of the fundamental classical laws of motion. It is the dynamic analogue to the principle of virtual work for applied forces in a static system and in fact is more general than Hamilton's principle, avoiding restriction to holonomic systems. A holonomic constraint depends only on the coordinates and time. It does not depend on the velocities. If the negative terms in accelerations are recognized as inertial forces, the statement of d'Alembert's principle becomes The total virtual work of the impressed forces plus the inertial forces vanishes for reversible displacements.

The principle states that the sum of the differences between the forces acting on a system of mass particles and the time derivatives of the momenta of the system itself along any virtual displacement consistent with the constraints of the system, is zero. Thus, in symbols d'Alembert's principle is written as following,

\footnotetext{
16 Source: bttp:/ / en.wikipedia.org/wiki/Bending

17 Source: http://en.wikipedia.org/wiki/Collision

18 Source: bttp://en.wikipedia.org/wiki/D\%27Alembert\%27s principle
} 
where:

$$
\sum_{i}\left(F_{i}-m_{i} a_{i}\right) \delta r_{i}=0
$$

- $\quad i \quad$ is an integer used to indicate a variable corresponding to a particle in the system,

- $\delta r_{i}$ is the virtual displacement of the $\mathrm{i}$-th particle, consistent with the constraints.

\section{TABLE OF NOMINATIONS}

\begin{tabular}{|l|c|l|c|}
\hline \multicolumn{1}{|c|}{ Quantity } & Nomination & \multicolumn{1}{c|}{ Quantity } & Nomination \\
\hline Length & $\mathrm{L}$ & Speed & $\mathrm{v}$ \\
\hline Density & $\rho$ & Constant of collision & $\mathrm{V}$ \\
\hline Young modulus & $\mathrm{E}$ & Angular acceleration & $\mathrm{B}$ \\
\hline Cross section & $\mathrm{A}$ & Acceleration & $\mathrm{a}$ \\
\hline Second moment of area & $\mathrm{I}$ & Pressure & $\mathrm{p}$ \\
\hline Stress & $\sigma$ & Force & $\mathrm{F}$ \\
\hline Angular speed & $\omega$ & Bending moment & $\mathrm{M}$ \\
\hline
\end{tabular}

\section{MODELING OF THE FEDER}

The goal of this section was to get the equations for the cross-section and the second moment of area, because the two parameters of the blade are changing along the length.

I created the ground model (for dynamic and stress analysis) for swords and feders in my Free Scholler thesis work ${ }^{19}$. In this analysis, I will only give a summary of the results and equations for feders, what I got before. The detailed computations, and theories can be found in the thesis work.

19 You can see it here: http://www.arsensis.hu/pdf/FS_RF.pdf, or the summary after this article 


\section{The original model}
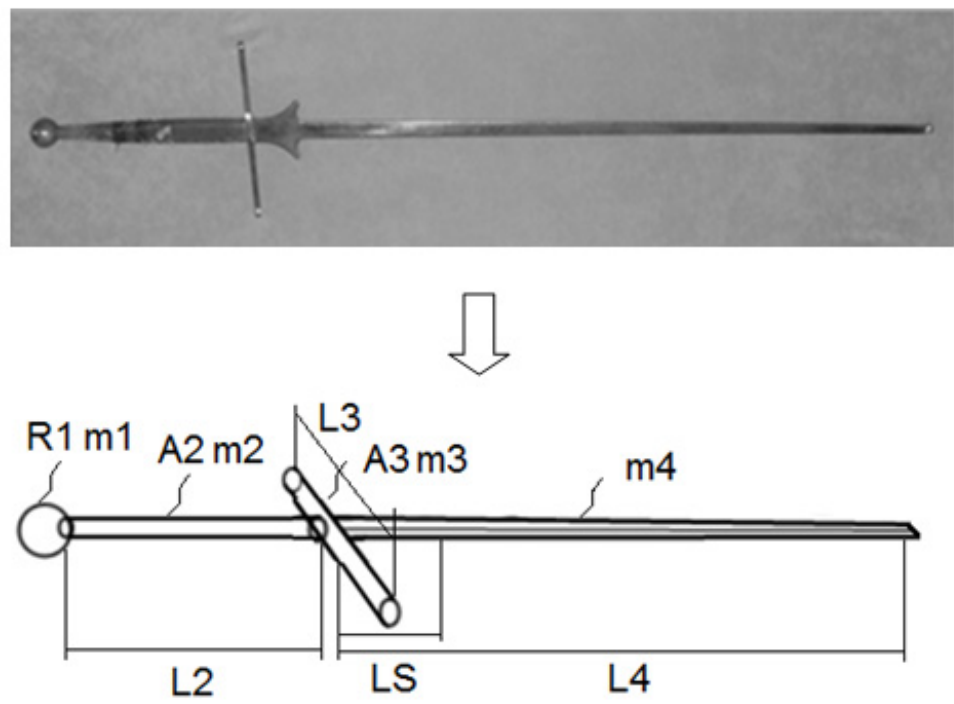

From the system above, I need only this ground data

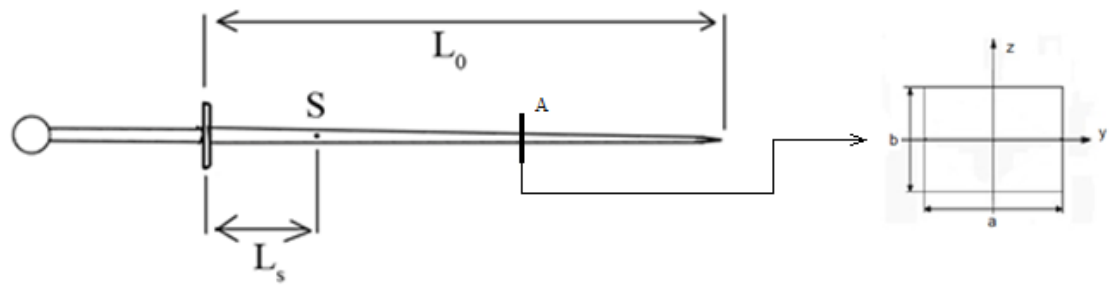

Pictures for ground data (model, cross section (Rectangle))

\begin{tabular}{|l|c|c|c|}
\hline \multicolumn{1}{|c|}{ Quantity } & Nomination & Value & Unit \\
\hline Length of the feder's blade & $\mathrm{L}_{0}$ & 0.95 & $\mathrm{~m}$ \\
\hline $\begin{array}{l}\text { The distance between the centre of gravity, } \\
\text { and the cross-guard }\end{array}$ & $\mathrm{L}_{\mathrm{S}}$ & 0.075 & $\mathrm{~m}$ \\
\hline Density of steel & $\rho_{\text {st }}$ & 7850 & $\mathrm{~kg} / \mathrm{m} 3$ \\
\hline Young modulus of steel & $\mathrm{E}_{s t}$ & 200 & $\mathrm{GPa}$ \\
\hline Braking stress in steel & $\sigma_{\max }$ & 400 & $\mathrm{MPa}$ \\
\hline $\begin{array}{l}\text { Length of the rectangle of the blade at the } \\
\text { cross-guard }\end{array}$ & $\mathrm{a}_{\mathrm{s}}$ & 27 & $\mathrm{~mm}$ \\
\hline $\begin{array}{l}\text { Width of the rectangle of the blade at the } \\
\text { cross-guard }\end{array}$ & $\mathrm{b}_{\mathrm{s}}$ & 5 & $\mathrm{~mm}$ \\
\hline $\begin{array}{l}\text { Length of the rectangle of the blade at the } \\
\text { end }\end{array}$ & $\mathrm{a}_{\mathrm{e}}$ & 11 & $\mathrm{~mm}$ \\
\hline Width of the rectangle of the blade at the end & $\mathrm{b}_{\mathrm{e}}$ & 1 & $\mathrm{~mm}$ \\
\hline
\end{tabular}


From this, I can write the equations for each length of the sides of the blade. I assume, that the sides are linearly decreasing their width from the start (cross-guard) to the end (top) of the blade.

The parametric equations for the feder :

$$
a(x)=\mathrm{a}_{\mathrm{s}}-\frac{\left(\mathrm{a}_{\mathrm{s}}-\mathrm{a}_{\mathrm{e}}\right) x}{\mathrm{~L}_{0}} ; \quad b(x)=\mathrm{b}_{\mathrm{s}}-\frac{\left(\mathrm{b}_{\mathrm{s}}-\mathrm{b}_{\mathrm{e}}\right) x}{\mathrm{~L}_{0}}
$$

These equations can be seen on the next figure (where the $\mathrm{x}$ axis is the symmetry axis of the blade )

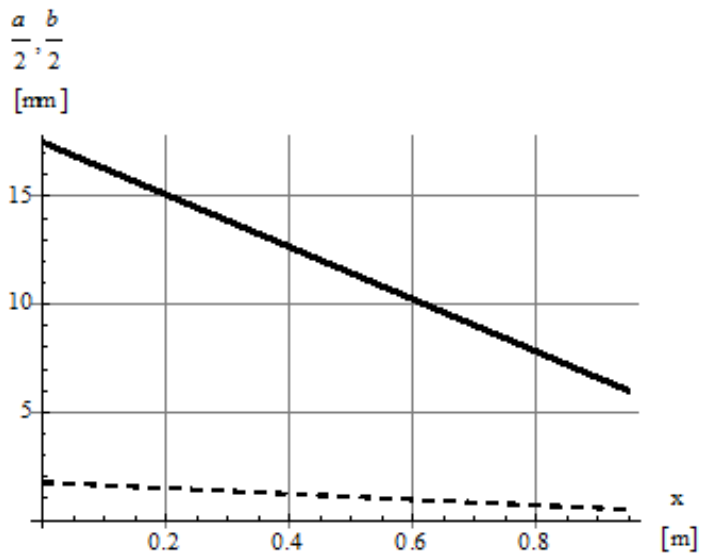

The half of the length and width $(a / 2, b / 2)$ of the cross section as function of the length of the blade

From the equations above, we can determine the function for the Cross section:

$$
A(x)=a(x) b(x)
$$

A

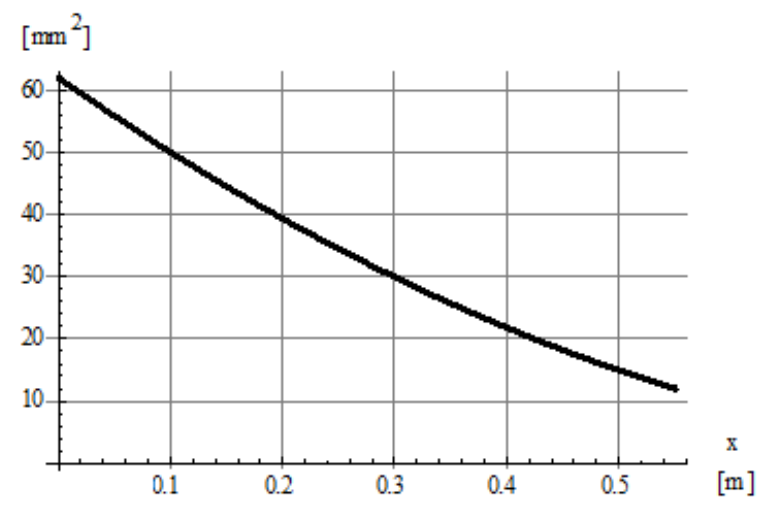




\section{The change of the area of cross-section along the length of the blade}

From the same equations $(a, b)$, we can calculate the function for the second moment of area:

$$
\mathrm{I}_{\mathrm{z}}(\mathrm{x})=\frac{a(x)^{3} b(x)}{12}
$$

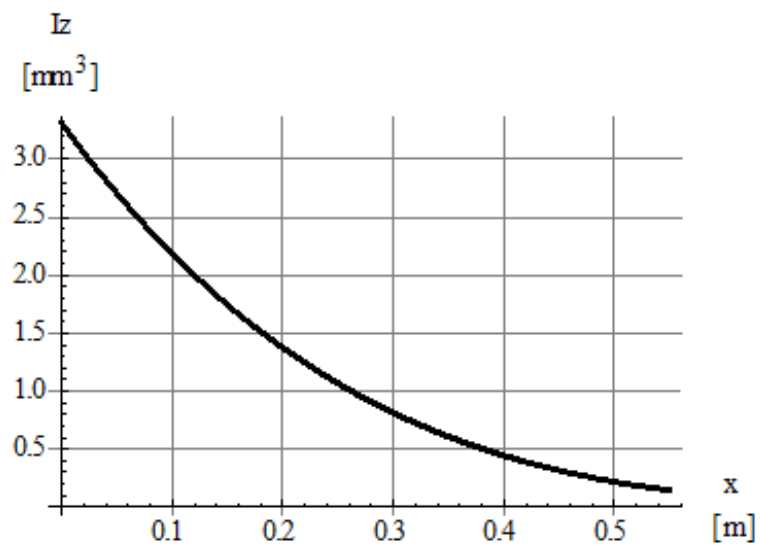

The change of the second moment of area along the length

\section{THE MODEL OF THE COLLISION}

I get the initial speed data from the dynamic analysis of my Free Scholar thesis work ${ }^{20}$

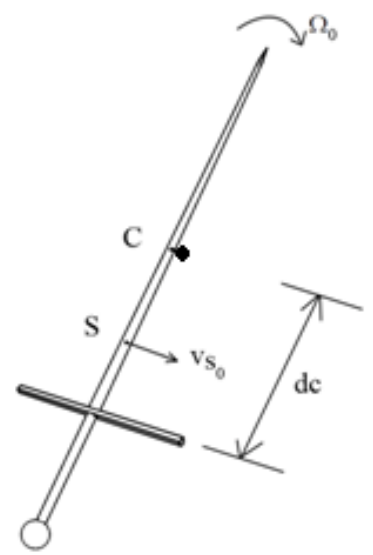

The initial data can be seen in this picture

\section{Quantity} Nomination 


\begin{tabular}{|l|l|l|c|}
\hline Initial rotational speed & $\omega_{0}$ & 10 & $\mathrm{rad} / \mathrm{s}$ \\
\hline Initial speed of the feder's centre of gravity & $\mathrm{v}_{0}$ & 8 & $\mathrm{~m} / \mathrm{s}$ \\
\hline
\end{tabular}

In the part of the collision, I will use a simplified model. I take the other feder as an infinite mass, which is not moving. To properly simulate the collision, I take the holding (where you grab the feder) of the sword as a joint. This means it can't move, but it can rotate So this simplification takes, that the collision becomes a fixed axis type. That can we see on the next picture.

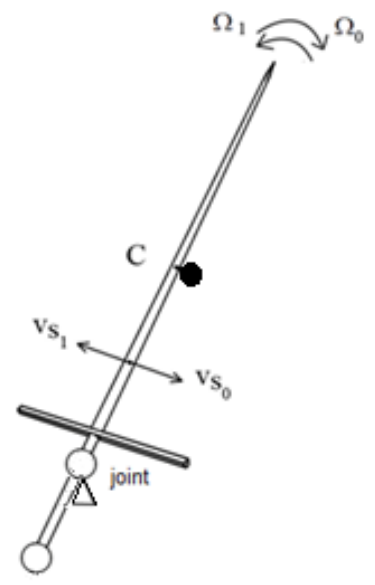

Model for collision

This means, the computation of the collision becomes very simple. So I can use the following forms:

$$
\begin{aligned}
\mathrm{v}_{1} & =-v \mathrm{v}_{0} \\
\omega_{1} & =-v \omega_{0}
\end{aligned}
$$

Where for the value of $v$ I choose 0.6. It is common by steel collisions. So we have the change of the velocity, and the angular velocity. From the video analysis, what I have done, I know the time, that elapses during the collision is less than 1 frame (the parameter of the video is 23.976 f.p.s.). From video analysis, I think it is 0.3 frame (as you can see on the next picture).

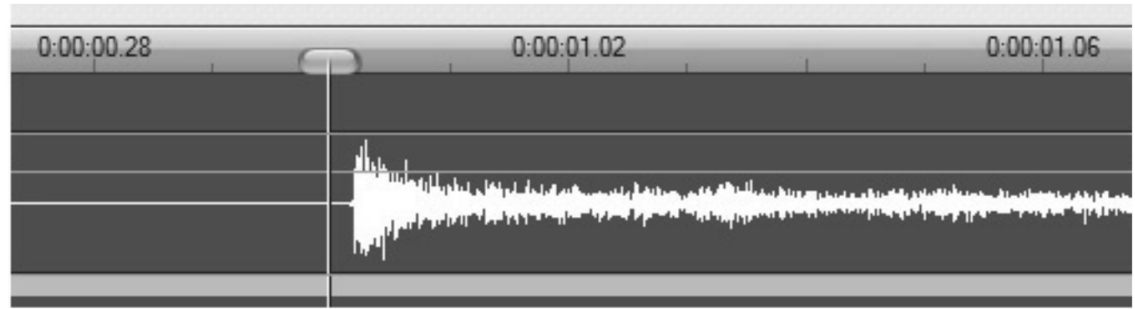

Sound track of two feders collision

$$
\Delta \mathrm{t} \approx 0.014 \mathrm{~s}
$$


From here, I can tell the acceleration state:

$$
\begin{aligned}
& \mathrm{a}_{\mathrm{c}}=\frac{\mathrm{v}_{1}-\mathrm{v}_{0}}{\Delta t} \\
& \beta_{\mathrm{c}}=\frac{\omega_{1}-\omega_{0}}{\Delta t}
\end{aligned}
$$

\begin{tabular}{|l|c|c|c|}
\hline \multicolumn{1}{|c|}{ Quantity } & Nomination & Value & Unit \\
\hline Angular acceleration from collision & $\mathrm{a}_{\mathrm{c}}$ & -922 & $\mathrm{rad} / \mathrm{s} 2$ \\
\hline $\begin{array}{l}\text { Acceleration of the feder's centre of gravity } \\
\text { from collision }\end{array}$ & $\beta_{\mathrm{c}}$ & -1152 & $\mathrm{~m} / \mathrm{s} 2$ \\
\hline
\end{tabular}

\section{Model after the collision}

At this time, I created a simplified model for the stress calculation. The modifications:

- I assume, that the cross section of the collision is not moving

- I get rid of the feder's other part (below the collision point)

So the blade can be modeled as a beam with one built-in end

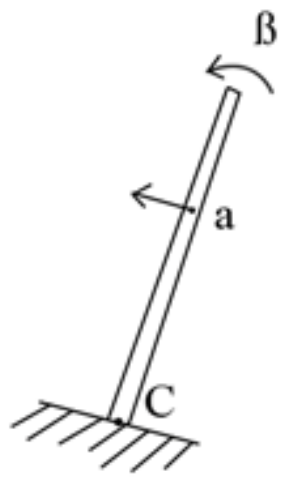

Model after collision

From here, I have to apply a new coordinate system. I push the function parallel to the $\mathrm{x}$ axis

$$
\begin{aligned}
& a(x)=a_{s}-\frac{\left(a_{s}-a_{e}\right)(x+d c)}{L_{0}} \\
& b(x)=b_{s}-\frac{\left(b_{s}-b_{e}\right)(x+d c)}{L_{0}}
\end{aligned}
$$

So with these new functions, I recalculate the function for the cross section and the second moment of area. 


\section{COMPUTING THE LOADING SYSTEM}

\section{Computing the accelerations along the modeled blade section}

Because of the rotation and the collision there will be a reaction force with $\mathrm{x}$ and $\mathrm{y}$ component at point $\mathrm{B}$ and there will be also a reaction torque. The effect of the breaking will be considered with the D'Alembert principle. For that we will need the acceleration field along the $\mathrm{x}$ axis, which can be calculated from the equation, that describes the acceleration state in rigid bodies:

$$
\underline{a_{B}}=\underline{a_{A}}+\underline{\beta} \times \underline{r_{A B}}+\underline{\omega} \times\left(\underline{\omega} \times \underline{r_{A B}}\right)
$$

From this, we get this two equations for the direction $x$ and $y$ :

$$
\begin{aligned}
& a(x)_{x}=\omega_{0}^{2} x \\
& a(x)_{y}=\mathrm{a}_{0}+\beta_{c} x
\end{aligned}
$$

From numerical calculation:

$$
a_{0}=-1296
$$

So applying the D'Alembert principle we get the intensity function of the distributed force:

$$
\begin{aligned}
& p(x)_{x}=\rho_{\mathrm{st}} a(x)_{x} A(x) \\
& p(x)_{y}=\rho_{\mathrm{st}} a(x)_{y} A(x)
\end{aligned}
$$

$\mathrm{px}$

$[\mathrm{N} / \mathrm{m}]$

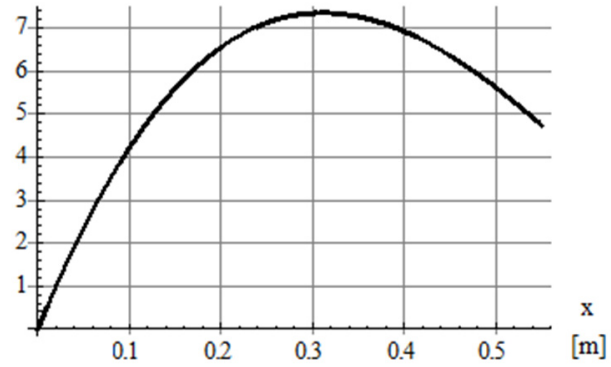

The distribution of the normal pressure

$$
\text { py }
$$

$[\mathrm{N} / \mathrm{m}]$

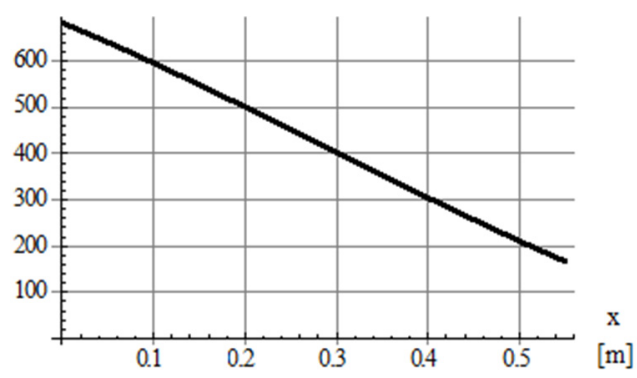

The distribution of the vertical pressure 
The reaction forces will withstand only this $p(x)$ along the blade. So the equilibrium equations based on the momentum and angular momentum theorems are given in these form:

$$
\begin{array}{ll}
\text { x direction: } & \mathrm{B}_{\mathrm{x}}=-\int_{0}^{\mathrm{L} 0-\mathrm{dc}} \mathrm{px} \mathrm{d} x \\
y \text { direction: } & \mathrm{B}_{\mathrm{y}}=-\int_{0}^{\mathrm{L} 0-\mathrm{dc}} \mathrm{py} \mathrm{d} x \\
z \text { direction: } & \mathrm{M}_{\mathrm{B}}=\int_{0}^{\mathrm{L} 0-\mathrm{dc}} \text { py } x \mathrm{~d} x
\end{array}
$$

The reaction forces and moment at point $\mathrm{B}$ is trivial from here, their values are:

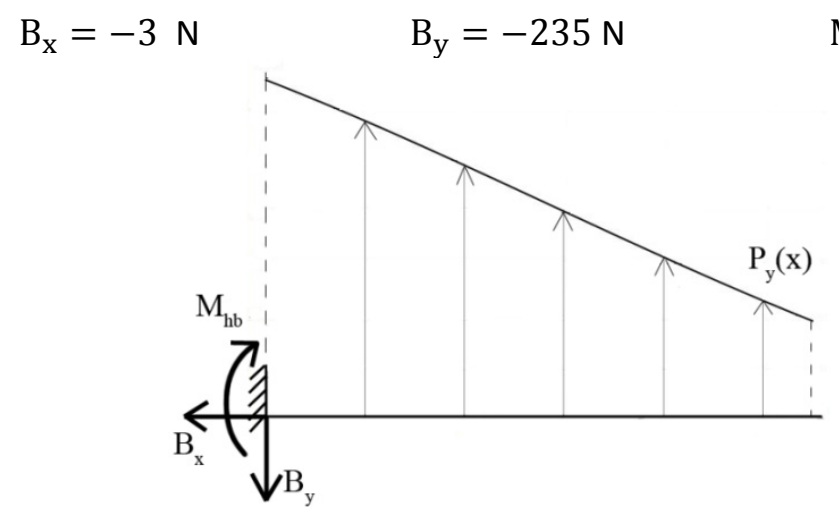

The Reaction forces, and the pressure on y axis

\section{The resultant functions}

We will have three stress resultant functions: the normal and the shear -force and the bending moment functions. The normal force function is shown in the next equation. The values are positives because in the blade the normal force is pulling.

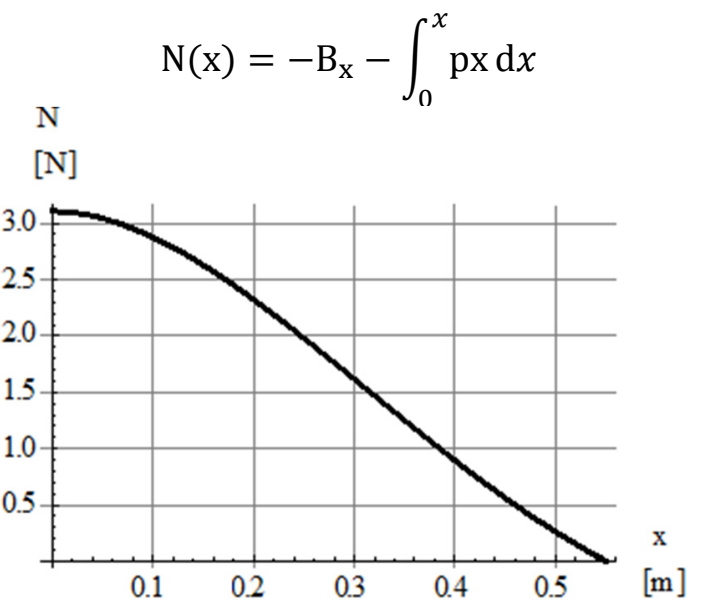

The distribution of the tensile pressure along the length of the model 
The shear-force is given in the next equation.

$$
\mathrm{V}(\mathrm{x})=\mathrm{B}_{\mathrm{y}}+\int_{0}^{x} \operatorname{pyd} x
$$

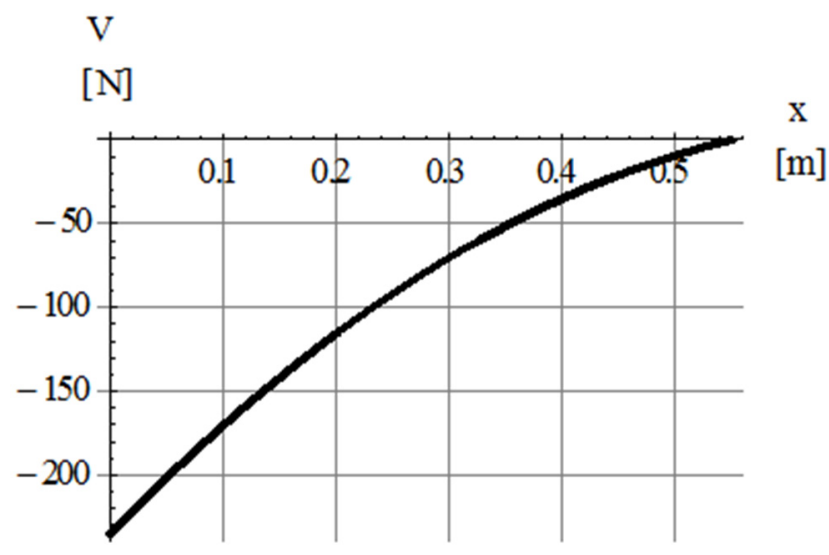

The distribution of the vertical pressure along the length of the model

Finally the formula of the bending moment function is given in the next formula

$$
\operatorname{Mh}(\mathrm{x})=\mathrm{M}_{\mathrm{B}}-\int_{0}^{x} \operatorname{py} x \mathrm{~d} x
$$

\section{Mh}
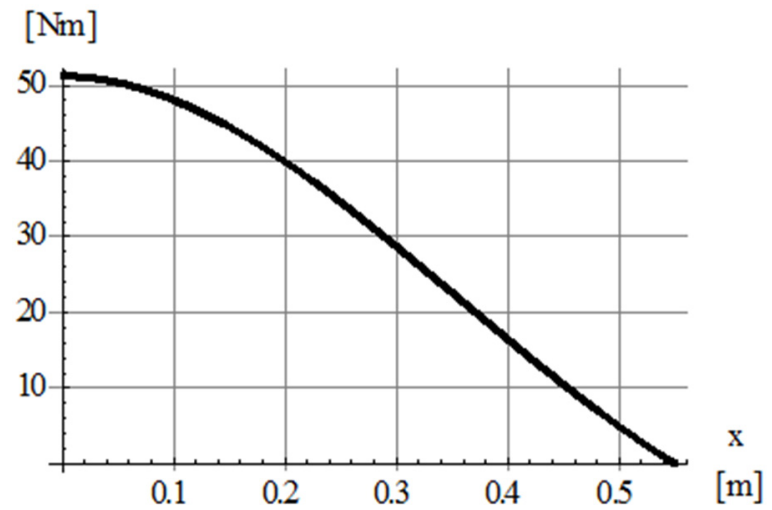

The distribution of the bending moment along the length of the model 


\section{STRESS AT THE CRITICAL CROSS SECTION}

From the calculations, what I have done, I can say, that the only part, which cause most of the stress is the bending moment. So we can use the following equation for stress calculation:

$$
\sigma(\mathrm{x})=\frac{\mathrm{Mh} \mathrm{a}}{2 \mathrm{I}_{\mathrm{z}}}
$$

\section{$\sigma \mathrm{x}$}

\section{[Mpa ]}

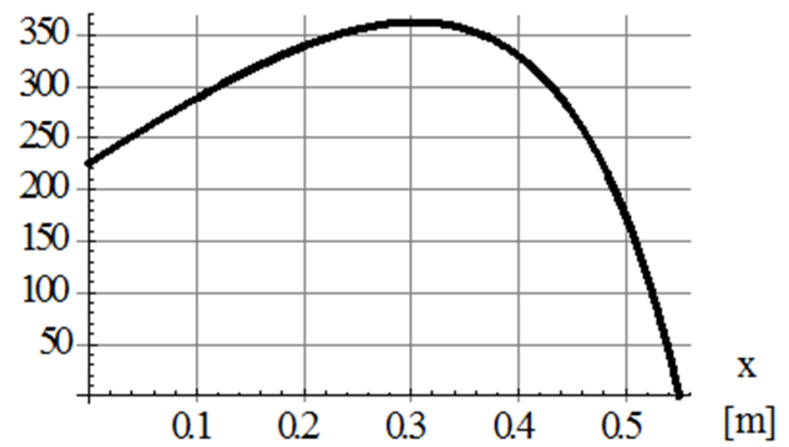

The distribution of the stress for the bending moment along the length of the model

From analytical calculation the maximal value, and the position is at:

$$
\sigma_{\text {max }}=362.7 \mathrm{MPa} \quad x=0.3 \mathrm{~m}
$$

The most important thing is, that the maximum is not at the point of collision! From the pressure curves above, most of the people think, that the maximum of the stress will be at point ' $\mathrm{C}$ '. But from here we can only tell, that this value is lower than the maximal stress, that the steel can take (400 MPa), it will not brake. (If there are some problems in the blade, it can easily brake)

\section{SMALL INJURY ON THE BLADE - STRESS INCREASING}

Let's suppose, that we did some fights with our feder. I think everyone did 'wrong hits' with it, and from these hits, we get some cavity on our feder. And lets suppose, that we have bad luck, and the point of this cavity is exactly the same place where maximal stress occurs. I do such type of perturbation on the feder's blade. I apply a function that can get such type of form. The function what I use:

$$
f(x)=-\frac{e^{-(4000(x-x 1))^{2}}}{1000}
$$

where $\mathrm{x} 1$ is the point, where maximal stress occurs. $(0.3 \mathrm{~m})$ 
So this will cause a $0.5 \mathrm{~mm}$ deep, $1 \mathrm{~mm}$ long hole on the feder's blade. For imagination, this means, that we lost $\sim 0.002$ gram steel from the feder (Less than $0.001 \%$ of the total mass)

\section{a inj}

[mm]

$\mathrm{X}$

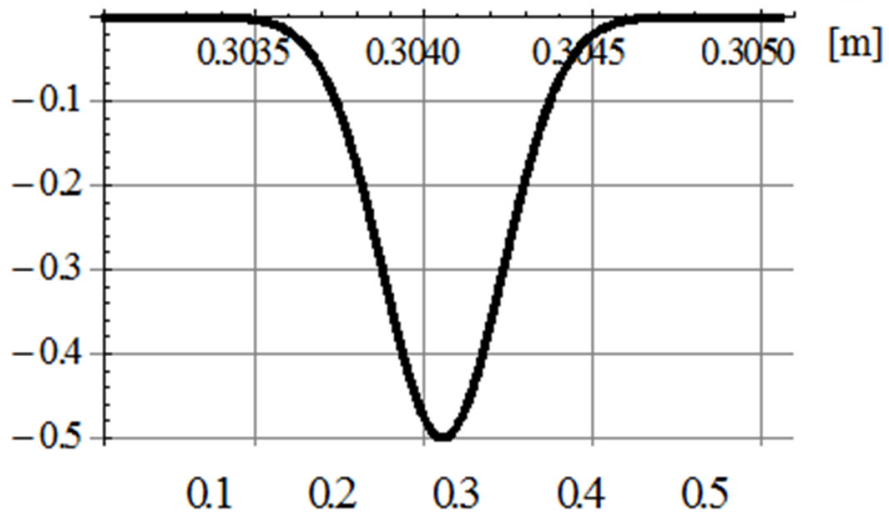

Depth of the bole along the coordinate $x$

From here, I recalculate the pressure and the stress functions, so at the end, I get the stress function. It looks like the same, as we have at the previous point, but we have a peek at the maximal stress value. With these peek, the maximal stress becomes:

$$
\sigma_{\max }=401 \mathrm{MPa}
$$

\section{$\sigma \times 1$}

[Mpa ]

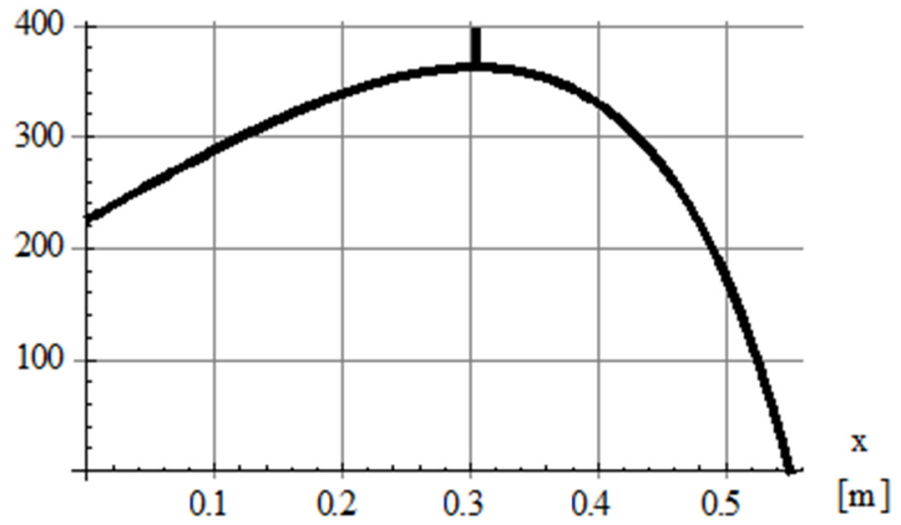

The distribution of the stress for the bending moment along the length of the model 
So the feder can be broken! And as we can see, that such little perturbation can cause problematic effects. It will raise $10 \%$ of the total stress value. But there is another important thing.If the hole's position is different from the point of the maximal stress then the effect is not so dramatic. If it is far enough, then the raise of the stress will be negligible.

\section{EXAMPLES FROM THE REAL LIFE}

I get some broken feders from my friends, Peter Regenyei, and Zoltan Berkes. I will show you this feders properties, and you can see the breaking points.

\begin{tabular}{|l|c|c|c|c|}
\hline Properties of the feders & $\mathbf{1 .}$ & $\mathbf{2 .}$ & $\mathbf{3 .}$ & $\mathbf{4 .}$ \\
\hline $\begin{array}{l}\text { Distance between the cross-guard and the point of the } \\
\text { brake [m] }\end{array}$ & 0.75 & 0.795 & 0.59 & 0.9 \\
\hline Distance between the point of collision the brake [m] & 0.35 & 0.395 & 0.19 & 0.5 \\
\hline Length of the rectangle of the blade at the cross-guard [m] & 0.035 & 0.027 & 0.027 & 0.027 \\
\hline Width of the rectangle of the blade at the cross-guard [m] & 0.005 & 0.005 & 0.005 & 0.005 \\
\hline Length of the rectangle of the blade at the end [m] & 0.015 & 0.011 & 0.011 & 0.011 \\
\hline Width of the rectangle of the blade at the end [m] & 0.0012 & 0.0015 & 0.0015 & 0.001 \\
\hline
\end{tabular}

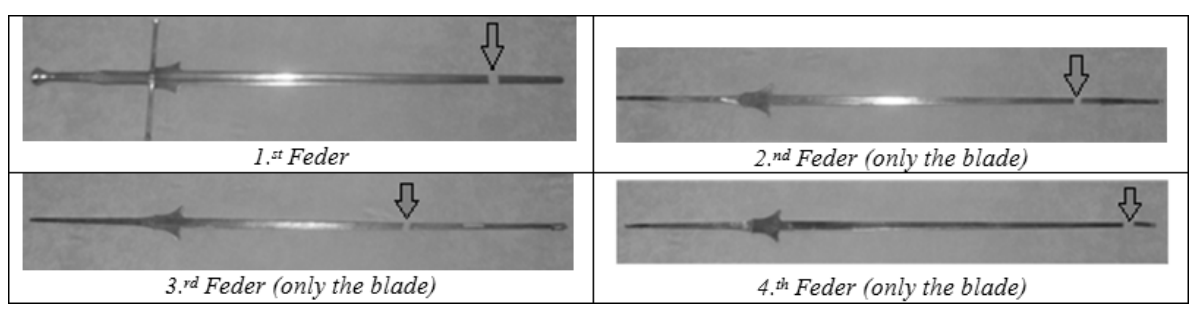

What we can see from this data and the pictures, that the maximal value of the stress will occur behind the point of the average collisions.

\section{A SOLUTION FOR THE PROBLEM}

I modified the functions, which describes the form of the cross sections width to a special form, where the width of the end of the blade is growing. Such type of form can be found in the Swiss National Museum, KZ 1030 (Fechtschwert)

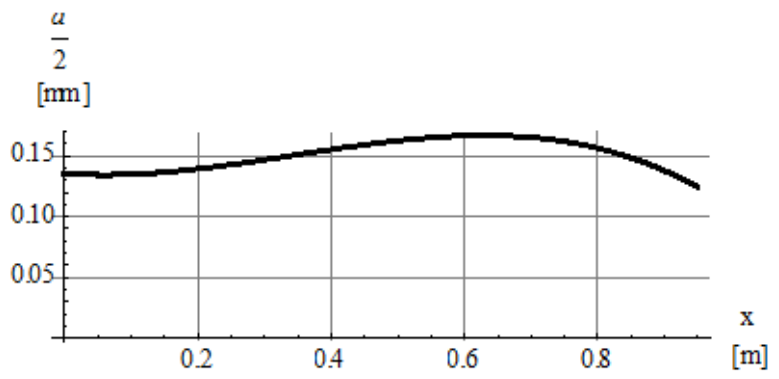

The function of the cross sections length 
From this form the stress resultant function becomes 'normal', so the maximal stress will occur at the point of collision.

$\sigma \mathrm{x}$

\section{[Mpa ]}

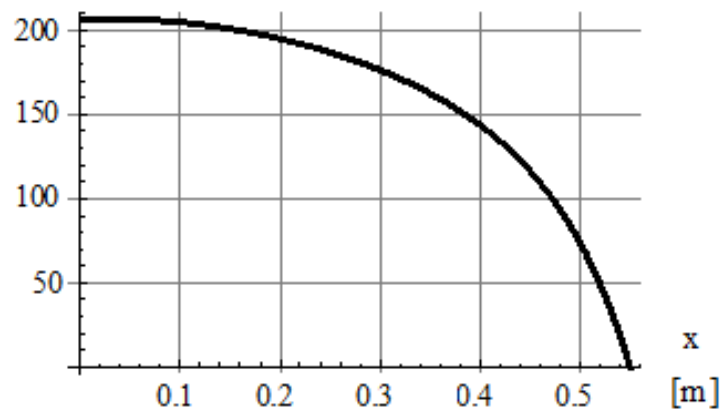

The stress resultant function

\section{SUMMARY}

I created a model for the feder, and after the computations of the collision, I created a new simplified model, to get the stress data which is caused by the acceleration field. From the stress curves, what I got I can tell the reason, why the feders broke in that way, what we saw. The most important result, what I got, that the maximal stress data was not in the point of collision, but it was between the point of collision and the end of the blade (when all of the parameters of the cross section (width, length) is decreasing to the end of the blade)! And if we have a small hole on the feder, than the maximal stress can increase $10 \%$ ! 